\title{
Addressing the Need for Context Awareness and Security Requirements in Wireless Body Area Networks
}

\author{
G. K. Ragesh and K. Baskaran
}

\begin{abstract}
Recent technical advancements in low-power integrated circuits, ultra low-power RF (radio frequency) technology, wireless communications and micro sensors allowed the realization of a futuristic health care system called Wireless Body Area Networks (WBANs). This new type of technology hold much promise for future patient health monitoring. A body area network wirelessly connects independent nodes (e.g. medical devices, earphones, sensors, actuators) attached to the body surface, implanted into tissues/body, or dispersed in the clothing for applications in home/health care, sports, entertainment, defense, ambient intelligence, pervasive computing and many other areas. These sensors offer promising applications in areas such as real time health monitoring, interactive gaming and consumer electronics. WBAN does not compel the patient to stay in the hospital thereby giving much physical mobility. This paper presents an overview on the various aspects of WBAN including the importance of context awareness in body area networks, applications of WBANs, major security requirements for data security and privacy in WBAN and various research challenges existing in this area
\end{abstract}

Index Terms-Wireless body area network, WBAN, body area network, real time health monitoring, bio sensors, context-awareness, data security and privacy.

\section{INTRODUCTION}

With the growing cost of healthcare and recent technological advancements in semi conductor technology allowed the realization of Wireless Body Area Networks (WBANs).This is one such emerging technology that has the potential to support remote patient monitoring in a reliable and cost effective manner thereby improving diagnostic monitoring, disease tracking and related health care procedures. Wireless Body Area Network (WBAN) consists of a number of inexpensive, lightweight, miniature sensors which could be located on the body surface, implanted in to the body tissues. These bio sensors provide promising applications in medial as well as non medical areas. This kind of a health care system provides much comfort for patients since sensors can be added without cables thus forming a wireless network between them. This WBAN technology enables doctors and other medical personnel to safely monitor the health status thus improving their quality of life in an affordable and efficient manner. Strategically placed wearable or implanted wireless sensor nodes consistently monitor the patient's vital signs, such as electrocardiogram (ECG), EEG and blood pressure; or

Manuscript received April 3, 2012; revised May 18, 2012.

G. K. Ragesh is with the Department of Computer Science and Engineering, Government College of Technology, Coimbatore, Tamil Nadu, India.

K. Baskaran is with the Department of Computer Science and Engineering, Government College of Technology, Coimbatore,Tamil Nadu,India important environmental parameters like temperature and humidity. The patient related data (gathered data) from all WBANs may ultimately be sent to a centralized healthcare repository for permanent records. Physicians can remotely access this data to assess the state of health of the patient. Additionally the patient can be alerted using SMS, alarm, or reminder messages.

In this article we present an overview of the state of the art in Wireless Body Area Networks. Our aim is to provide a better understanding of security related research issues in this emerging field. The remainder of this paper is organized as follows. First, WBAN system architecture is discussed in Section 2. Next, need for context awareness in BANs is discussed in Section 3. Section 4 deals with the WBAN applications. Section 5 discusses the various research challenges. Security requirements of WBAN are treated in section 6.Finally section 7 concludes the paper.

\section{WBAN SYSTEM ARCHITECTURE}

WBAN work through a process of data being transmitted from an implanted device to an external device. The sensor that is implanted inside the patient's body wirelessly interacts with other sensors and actuators. An actuator is the mechanism by which an agent acts upon an environment. The agent can be either an artificial intelligent agent or any other autonomous being.An autonomous being can be a human being or an animal. The Body Area Network functions by passing data from each sensor to a main station. The main station then fuses the data passed from each of the sensors and it is then sent to a recipient via the internet. The sensors of a Body Area Network are extremely compact and complex in design. The fact that the sensors are so minute means that the patients will be able to lead a normal life, as the sensor devices are very unobtrusive. All sensors produced will contain the same basic elements such as a power supply and wireless transceiver as well as a control mechanism, a sensor and the casing that will hold all of the components together. The sensors will be designed in a way that allows them to be self-governing for the entire lifetime. The communication in WBAN is a form of many to one communication where all traffic end up at a single point in the network. As WBAN assumed to be a connected network, nodes can either make a direct connection with the sink or it can rely on other nodes to make a contact. To simplify the analysis and to bring high energy efficiency most of the research works assume symmetric links and multi-hop topologies in WBAN, although this may not be the case in reality.

\section{NEED FOR CONTEXT AWARENESS IN BANS}

Context is defined as any information that can be used to 
characterize the situation of an entity, where an entity can be a person, place or physical object [14].This kind of technique is used to detect the internal or external state of a user. Context-aware computing describes the situation of a wearable or mobile computer being aware of the user's state and surroundings, and modifying its behavior based on this information [15].Context-awareness plays an important role in body area networks because it interprets various physical and biochemical signals coming from body sensor networks by considering the present state of the user and the state of the environment. Many of the algorithms developed in context aware sensing have different characteristics and accomplish different goals. But the ultimate goal of any context aware sensing algorithms is to achieve long term pervasive health care monitoring. Most of these approaches are combined with one another to achieve the context from the environment. The initial step of these approaches is to gather the low-level sensor readings from all sensor nodes and the data is typically clustered into subgroups by means of a clustering algorithm [16].

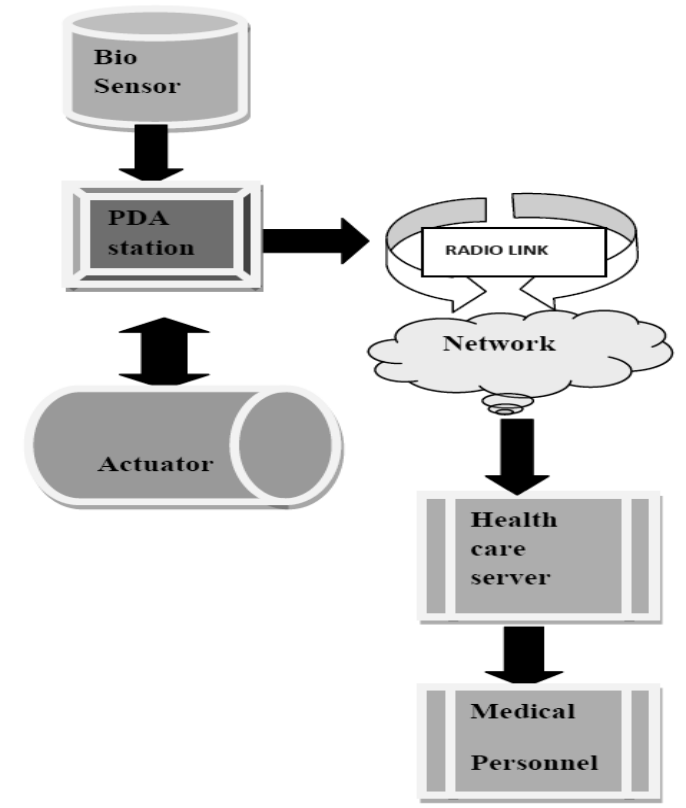

Fig. 1. Data flow in a typical medical BAN.

\section{A. Existing Context Aware Mechanisms}

A number of context aware mechanisms have been proposed for a variety of sensing applications. Some of them are described here. Tapia et al proposed the use of Naïve Bayesian classifier for activity recognition [17]. Kautz et al. introduced the use of hierarchical hidden semi-Markov models (HHSMMs) for tracking daily activities [18]. Laerhoven and Cakmakci described an integrated approach with SOM and K nearest neighbour for classifying different activities [19]. For arrhythmia detection, Lagerholm et al. proposed an integrated method for clustering QRS complexes by the use of self-organising maps (SOM) [20]. Simelius et al. introduced a SOM based spatiotemporal analysis for detecting abnormal ventricular activation [21]. Gao et al. proposed a neural network classifier based on a Bayesian framework for identifying arrhythmias [22]. Thiemjarus et al. proposed a feature selection technique for identifying relevant features or sensors for determining the optimal setup of the sensors in terms of location and channel selection [23].

\section{APPLICATIONS OF BANS}

Due to the diverse components that can be connected and integrated, body area networks will be able to provide various functions in healthcare, emergency, work, research, lifestyle, sports, or military.

\section{A. Medical Applications}

BANs can provide interfaces for diagnostics, for remote monitoring of human physiological data, for administration of drugs in hospitals and as an aid to rehabilitation. In the future it will be possible to monitor patients continuously and give the necessary medication whether they are at home, in a hospital or elsewhere. Patients will no longer need to be connected to large machines in order to be monitored.

\section{B. Lifestyle and Sports}

BANs enable new services and functions for wireless body-centric networks including wearable entertainment system (e.g., music entertainment), navigation support in the car or while walking, museum or city guide, heart rate and performance monitoring in sports, infant monitoring, wireless cash card (e.g., display of recent transactions and checking of balance, etc).

\section{Military Applications}

The opportunities for using BANs in the military are numerous. Some of the military applications for BANs include monitoring health, location, temperature and hydration levels. A battle dress uniform integrated with a BAN may become a wearable electronic network that connects devices such as life support sensors, cameras, RF and personal PDAs, health monitoring GPS, and transports data to and from the soldier's wearable computer. The network could perform functions such as chemical detection, identification to prevent casualties from friendly fire and monitoring of a soldier's physiological condition. Calling for support, his radio sends and receives signals with an antenna blended into his uniform. As a result, BANs provide new opportunities for battlefield lethality and survivability.

\section{RESEARCH CHALLENGES}

A lot of research has investigated to physical layer. At the beginning of WBAN research a number of authors proposed Ultra Wide Band (UWB) as a physical layer for WBANs.UWB has the advantage of low energy consumption, good co-operation with existing wireless networks and a range large enough to support the entire body. Due to standardization issues and difficulties delivering the very high speeds UWB does not progress well. As opposed to the wide bands proposed by UWB, other researchers propose the small, Industrial, Scientific and Medical (ISM) bands of the IEEE 802.15.4 andIEEE802.15.6.Current most working WBAN prototypes are based on ISM bands. A number of WBAN specific MAC protocols exist. These can be divided in to single hop and multi hop protocols. The latter refers to the protocols which are optimized for multi hop topologies. The first 
protocols were designed based on a single hop topology. An example for this is Heart Beat driven MAC (H-MAC), which uses the heart beat to synchronize nodes. The protocol is specifically designed for WBANs; however traffic adaptations is not possible. Few other protocols have been developed usually IEEE 802.15.4 is preferred. Because of the dynamic nature, ad hoc network protocol could also be considered as WBAN protocols. Ad hoc network protocols are based on always -on radios, which matters their application to WBAN unfeasible. A number of routing protocols for sensor and ad hoc networks could be considered good candidates for WBANs. The WSN protocols will focus on networks of a much larger scale while ad hoc network routing protocols will assume nodes with a larger battery and an always on radio. The sensors in a WBAN are located in or on the human body which can be in motion. This challenge for WBAN is rarely available for WSNs. Thus the WBAN must be robust against the high probable network topology changes. In addition, biological variation and complexity cause a more variable structure. Because of the special features of the environment in which the WBAN operates (human body) the data loss is more significant. The signals of the sensors, specially the implanted ones, are considerably attenuated because the propagation of the waves takes place in or on a very lossy medium. Proprietary mechanisms may be required to ensure the QoS and real time data interrogation capabilities. The small size of the WBAN sensors severely affects the power resources of the devices.

\section{SECURITY REQUIREMENTS}

The use of wireless technology, especially to deliver health care, also brings with it a host of concerns about security and privacy. The security mechanism plays a significant role. The security mechanism of the system is responsible for providing the following security services on specified biomedical data when requested to do so by the applications.

- Data Encryption: the data is encrypted so that it is not disclosed whilst in transit. Data encryption service provides confidentiality against eavesdropping attacks.

- Data Integrality: Data integrality service consists of data integrity and data origin authentication. Proper data integrity mechanisms at the $\mathrm{BN}$ and the $\mathrm{BNC}$ ensure that the received data is not altered by an adversary

- Freshness Protection: Data freshness ensures that the data frames are in order and are not reused.

- Authentication: an efficient method against impersonation attacks.

TABLE I:SECURITY REQUIREMENTS FOR PATIEN-RELATED DATA IN WBAN

\begin{tabular}{|l|l|}
\hline Major security requirements & \multicolumn{1}{|c|}{ Description } \\
\hline Data Confidentiality & $\begin{array}{l}\text { Patient- related data should be kept confidential and robust against node } \\
\text { compromise and user collusion. }\end{array}$ \\
\hline Data Integrity & Patient- related data must not be modified illegally. \\
\hline Data Dependability & Patient -related data should be readily retrievable when node failure happens. \\
\hline Data Access control & $\begin{array}{l}\text { To prevent unauthorized access to patient related data WBAN needs a fine-grained } \\
\text { data access control policy. }\end{array}$ \\
\hline Data Accountability & $\begin{array}{l}\text { If a WBAN user abuses other user's privileges to carry out unauthorized actions. } \\
\text { then those users should be indentified and held accountable. }\end{array}$ \\
\hline Revocability & $\begin{array}{l}\text { If a WBAN user is identified as compromised or behave maliciously then the } \\
\text { privileges of that user should be deprived in time. }\end{array}$ \\
\hline Authentication & $\begin{array}{l}\text { Only authenticated users can send data in WBANs and all other outside injection } \\
\text { of data must be prevented. }\end{array}$ \\
\hline
\end{tabular}

\section{CONCLUSION AND FUTURE WORK}

In this paper various key aspects of WBAN including system architecture, importance of context awareness in BANs, WBAN applications are outlined. Also discussed security requirements and research challenges/social issues related to WBAN application. There are many challenges that still need to be addressed on high bandwidth and energy efficient communication protocols, interoperability between BANs and the design of successful applications. Future work will be concentrating on the design of a light weight flexible cryptographic mechanism which will carefully optimize security, safety and usability. We hope this paper will inspire practical designs of cryptographic enforced, context aware, dependable and privacy enhanced WBANs.

\section{REFERENCES}

[1] M. Patel and J. F. Wang, "Applications, challenges and prospective in emerging body area networking technologies," IEEE Wireless Communications, 1536-1284, 2010 IEEE

[2] M. Li and W. Lou, "Data security and privacy in wireless body area networks," IEEE Wireless Communications, 1536-1284, February 2010 .

[3] E. Jovanov et al, "A wireless body area network of intelligent motion sensors for computer assisted physical rehabilitation," J. NeuroEng. Rehab. vol. 2, no. 6, 2005.

[4] X. Huangy, H. Shany, and X. Shen, "On energy efficiency of cooperative communications in wireless body area network," 2011 IEEE.

[5] S. Rashwand, J. Misic, and H. Khazaei, "Performance Analysis of IEEE 802.15.6 under saturation condition and error prone channel," 2011 IEEE-WCNC-NETWORK

[6] S. Marinkovic and E. Popovici, "Nano- Power Wake-Up Radio Circuit for Wireless Body Area Networks," 2011 IEEE.

[7] J. K, V. R.SarmaDhulipala and R. M. C. Sekaran, "A WSN based frame work for human health monitoring". 
[8] Y. S. Kim and I. Y. Cho, "Wearable ECG monitor- evaluation and experimental analysis," 2011 IEEE.

[9] M. Barua, M. S. Alam, X. H Liang, and X. M (Sherman) Shen, "Secure and quality of service assurance scheduling scheme for WBAN with application to ehealth," IEEE WCNC 2011 -Network.

[10] X. Huang, H. G. Shan, and X. S. Shen, "On energy efficiency of cooperative communications in wireless body area networks, " IEEE WCNC 2011 -Network.

[11] D. Cypher, N. Chevrollier, N. Montavont, and N. Golmie, "Prevailing over wires in healthcare environments: benefits and challenges," IEEE Communications Magazine, vol. 44, no. 4, pp. 56-63, Apr. 2006

[12] S. Drude-"Requirements and application scenarios for body area networks," in Mobile and Wireless Communications Summit, 16th IST, Budapest, Hungary, Jul. 2007, pp. 1-5, 2007.

[13] G. K Ragesh and K. Baskaran, "A Survey on Futuristic Health Care System: WBANs", Elsevier Ltd. 1877-7058 (C) 2011 [Online]. Available: www.sciencedirect.com -Procedia Engineering 30, pp. 889 - 896, 2012.

[14] M. contaldo et al, "A 2.4-GHz BAW-Based transceiver for wireless body area networks" IEEE Transactions on bio medical circuits and systems, 2010.

[15] A. K. Dey, D. Salber, G. D. Abowd, and M. Futakawa, "The conference assistant: Combining context-awareness with wearable computing," In Proceedings of the 3rd. International Symposium on Wearable Computers, pp. 21-28, 1999

[16] A. Krause, D. Siewiorek, A. Smailagic, and J. Farringdon, "Unsupervised, dynamic identification of physiological and activity context in wearable computing," In Proceedings of the 7th International Symposium on Wearable Computers, pp.88-97, 2003.

[17] B. Fritzke, "Some competitive learning methods. Artificial intelligence institute, dresden university of technology," Tapia E. M., Intille S. S. and Larson K. 2004, in Proceedings of the 2nd International Conference on Pervasive Computing, pp. 158-175,1997.

[18] H. Kautz, O. Etziono, D. Fox, and D. Weld, 2003.Technical report CSE-020AC-01, University of Washington, Dept of Comp. Sci. and Eng.Laerhoven K. Van and Cakmakci O. 2000, in Proceedings of the Fourth International Symposium on Wearable Computers (ISWC 2000)

[19] M. Lagerholm, C. Peterson, G. Braccini, L. Edenbrandt, and L. Sornmo, IEEE Transactions on Biomedical Engineering, vol, 47 no. 7, pp. 838-848, 2000.

[20] K. Simelius, M. Stenroos, L. Reinhardt, J. Nenonen, I. Tierala, M. Makijarvi, L. Toivonen, and T. Katila, Physiological Measurement, vol. 24 , no. 3, pp. 805-816, 2003

[21] D. Gao, M. Madden, M. Schukat, D. Chambers, and G. Lyons, 2004. In Proceedings of the 24th SGAI International Conference on Innovative Techniques and Applications of Artificial Intelligence, vAI 2004.

[22] S. Thiemjarus, B. P. L. Lo, K.V. Laerhoven, and G. Z. Yang, In Proceedings of the 1st International Workshop on Wearable and Implantable Body Sensor Networks, 2004.

[23] S. Thiemjarus, B. P. L. Lo, K. V. Laerhoven, and G. Z. Yang Proceedings of the 1st International Workshop on Wearable and Implantable Body Sensor Networks, 2004. 